\title{
Self-diffusion in liquid gallium and hard sphere model
}

\author{
Nikolay Blagoveshchenskii ${ }^{2}$, Arkady Novikov ${ }^{2}$ Alexander Puchkov ${ }^{2}$, Vadim Savostin $^{2}$ and Oleg Sobolev ${ }^{1, \text { a }}$ \\ ${ }^{1}$ Institute of Physical Chemistry, Georg-August-University of Göttingen, Tammannstrasse 6, 37077 Göttingen, \\ Germany \\ ${ }^{2}$ State Scientific Center of the Russian Federation, Institute for Physics and Power Engineering, Bondarenko Sq.1, \\ Obninsk 249033, Russia
}

\begin{abstract}
Incoherent and coherent components of quasielastic neutron scattering have been studied in the temperature range of $\mathrm{T}=313 \mathrm{~K}-793 \mathrm{~K}$ aiming to explore the applicability limits of the hard-sphere approach for the microscopic dynamics of liquid gallium, which is usually considered as a non-hard-sphere system. It was found that the non-hard-sphere effects come into play at the distances shorter than the average interatomic distance. The longer range diffusive dynamics of liquid Ga is dominated by the repulsive forces between the atoms.
\end{abstract}

\section{Introduction}

Liquid gallium $(\mathrm{Ga})$ has singular structural and electronic properties indicating that it cannot be considered as a hard-sphere liquid. Ga does not crystallize in any of the simple crystal structures demonstrating an extremely rich structural polymorphism in the solid phase characterized by the competition between metallic and covalent bonding. Neutron scattering experiments also demonstrate that structural and dynamical properties of liquid Ga strongly deviate from that of the simple liquid [1-4].

On the other hand there are experimental results $[3,5]$ showing the hard-sphere model [6-13], which describes with a good accuracy the diffusive microscopic dynamics of the simple liquids, can be also applicable in the case of liquid $\mathrm{Ga}$. The coherent quasielastic linewidths measured with synchrotron [5] and neutron scattering [3] were successfully fitted within the framework of the extended heat mode approximation of Enskog's kinetic theory [7, 8]. However, a good agreement with the experimental data was demonstrated only for the temperature near the melting point and employing an "effective" hard-sphere diameter that was larger than the one related to the structure factor $S(Q)$ peak maximum.

In this paper we are presenting the more detailed analysis aiming to explore the applicability limits of the hard-sphere approach for the microscopic dynamics of liquid Ga. For this purpose, we used our quasielastic neutron scattering (QENS) data measured in a wide temperature range. The details of the short range dynamic were obtained from coherent scattering near the maximum of $S(Q)$, whereas the long range single particle motions were studied by the analysis of incoherent scattering measured at low $Q$ values.

\footnotetext{
${ }^{a}$ Corresponding author: sobolev38@gmail.com
}

\section{Models and methods}

Enskog kinetic theory for a dense hard-sphere system provides the following formula for the diffusion coefficient [5]:

$$
D_{E}=\frac{1}{16} \frac{\sigma}{\varphi} \sqrt{\frac{\pi k_{B} T}{m}} \frac{(1-\varphi)^{3}}{(1-\varphi / 2)},
$$

where $\varphi=\pi \rho \sigma^{3} / 6$ is a packing fraction, $\sigma, \rho$ and $\mathrm{m}$ are the hard sphere diameter, the atomic density and the mass of the particle correspondingly.

Enskog theory is based on the molecular chaos approximation. However in a real system there are correlated motions. The molecular dynamics simulations provide the ratio between the real hard sphere diffusion coefficient and predicted by Enskog theory, so called Alder-Wainwright (AW) correction [8-12]. The AW correction is a function of the reduced density

$$
V_{0} / V=\rho \sigma^{3} / \sqrt{2}
$$

and can be approximated by the following formula [12]:

$$
\begin{aligned}
D=D_{E} & {\left[1+0.05403\left(\frac{V_{0}}{V}\right)+6.3556\left(\frac{V_{0}}{V}\right)^{2}\right.} \\
& \left.-10.9425\left(\frac{V_{0}}{V}\right)^{3}\right] .
\end{aligned}
$$

In fact, formula (2) is an empirical correction for the correlated motions that are not taken in to account by Enskog's theory.

The deviation of the pair potential repulsive part from the hard-sphere form can be taken into account supposing the temperature dependence of the sphere diameter. A very simple semi-empirical expression for such a correction was proposed in Ref. [13] for liquid metals:

$$
\sigma_{T}=\sigma_{m}\left[1-0.112\left(T / T_{m}\right)^{1 / 2}\right] /(1-0.112) .
$$


The diameter at the meting point $\sigma_{\mathrm{m}}$ is calculated as follows:

$$
\sigma_{m}=\left[6 \times 0.472 M / \pi \rho_{m} N_{0}\right]^{1 / 3},
$$

where $M$ is the atomic weight, $N_{0}$ is Avogadro number and $\rho_{\mathrm{m}}$ is the mass density at the melting point.

The diffusion coefficient can be obtained from the incoherent QENS data using the simple diffusion model, which is valid at the low momentum transfer limit $\mathrm{Q} \rightarrow 0$ that gives the following expression for the quasielastic linewidth:

$$
\Delta \omega_{\text {incoh }} \approx D Q^{2} \text {. }
$$

Using the hard sphere approach the quasielastic coherent width can be written as follows [7,8]:

$$
\Delta \omega_{c o h}=\frac{D_{E} Q^{2}}{S(Q)} d(Q \sigma),
$$

where

$$
d(Q \sigma)=\frac{1}{1-j_{0}(Q \sigma)+2 j_{2}(Q \sigma)},
$$

$j_{0}$ and $j_{1}$ are the first two even order Bessel spherical functions.

\section{Experiment and data treatment}

The neutron measurements were carried out at the timeof-flight spectrometer DIN-2PI installed at the pulsed fast reactor IBR-2 (Frank Laboratory of Neutron Physics, JINR, Dubna) [14]. The initial neutron energy $E_{0}=$ $7.65 \mathrm{meV}$ provided the elastic peak resolution $\Delta E_{0} \sim$ $0.5 \mathrm{meV}$ and the range of neutron wave-vector transfer $0.3 \AA^{-1}<Q<2.8 \AA^{-1}$. The choice of rather high $E_{0}$. was dictated by the necessity to reach $Q$ values corresponding to the maximum of the $S(Q)$. Thus the resolution was good enough for the coherent data analysis, but not quite optimal for the incoherent data.

The sample consisted of nineteen molybdenum tubes with outer diameter of $6 \mathrm{~mm}, 0.25 \mathrm{~mm}$ of wall thickness, and $110 \mathrm{~mm}$ in height, filled by pure gallium and uniformly distributed to form the cylinder of $70 \mathrm{~mm}$ diameter. The neutron scattering spectra were measured at $313,433,553$, 673 , and $793 \mathrm{~K}$.

After corrections for detectors efficiency, attenuation, subtraction of the scattering by container, the experimental spectra were transformed to the scattering functions $S(Q, \omega)$ at $Q=$ const .

The incoherent and coherent QENS components were extracted from the data recorded in $Q$ regions $0.4 \AA^{-1}<$ $\mathrm{Q}<1.5 \AA^{-1}$ and $2.2 \AA^{-1}<Q<2.8 \AA^{-1}$ correspondingly. This was done using a special computer code that calculates partial components of the double-differential scattering cross-section based on a model or experimental information available in literature and taking into account multiple scattering and instrumental resolution. The details of the procedure can be found elsewhere $[15,16]$. These corrections were almost negligible in the case of the coherent scattering near the maximum of $S(Q)$, but they were very important for the incoherent component, mainly,
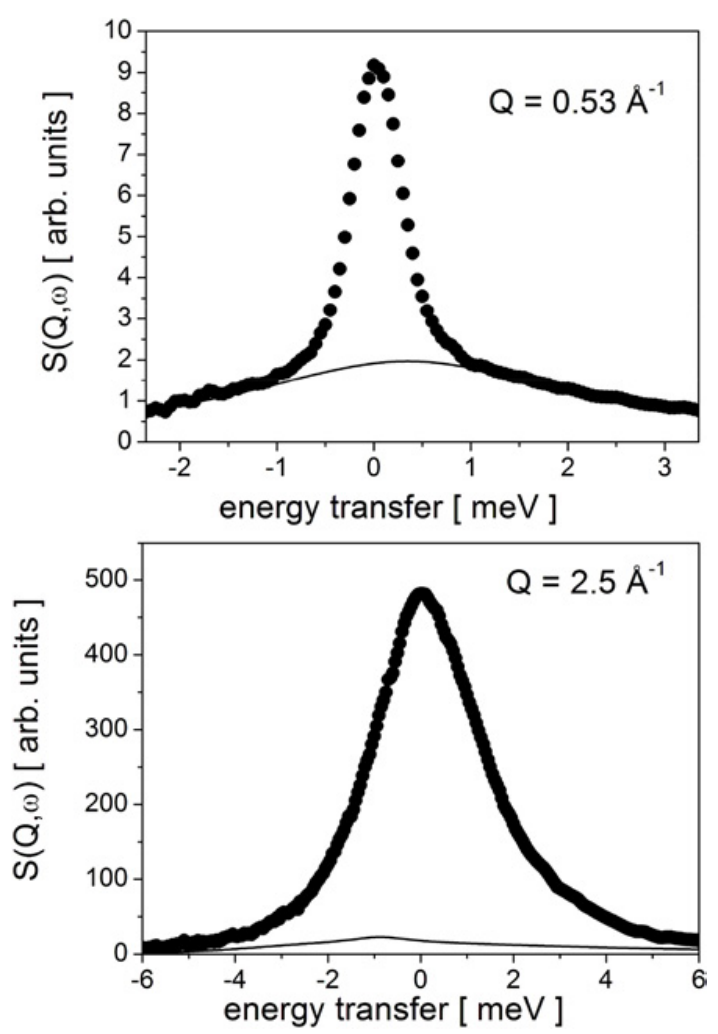

Figure 1. Neutron scattering spectra measured at $\mathrm{T}=673 \mathrm{~K}$ (points) and calculated components (lines). The line for $Q=$ $0.53 \AA^{-1}$ represents mainly multiple scattering (inelastic and coherent quasielastic scattering are almost negligible). The line for $Q=2.5 \AA^{-1}$ is the sum of multiple, inelastic and incoherent quasielastic scattering.

due to multiple scattering, since $S(Q)$ shows low values in the corresponding $Q$ region (Fig. 1).

The extracted QENS components were fitted by Lorentz functions convoluted with the resolution function of the spectrometer. The aim of this fitting procedure was to obtain the QENS line widths.

\section{Results and discussion}

It should be noted that the analysis of the incoherent QENS component was not very precise due to rather low instrumental resolution. The obtained incoherent full widths at half maximum $(F W H M=2 \Delta \omega(Q))$ are shown in Fig. 2 as a function of $Q^{2}$. Model (5) was used for fitting the data. The widths measured at all temperatures, except $\mathrm{T}=793 \mathrm{~K}$, show roughly a linear dependence on $Q^{2}$. The points corresponding to $\mathrm{T}=793 \mathrm{~K}$ deviate strongly from the linear law. Figure 3 presents the results of fitting, the self-diffusion coefficients together with the values obtained from the viscosity data using Stocks-Einstein relation [17].

Model (2) shows a reasonable agreement with the experimental data, but only if the hard-sphere diameter $\sigma$ is supposed to be temperature dependent according to Eq. (3). This result is very important for the analysis of our coherent QENS data.

The calculated $\sigma_{T}$ used for our data analysis is shown in Fig. 4. For comparison, the $\sigma_{T}$ values for liquid 


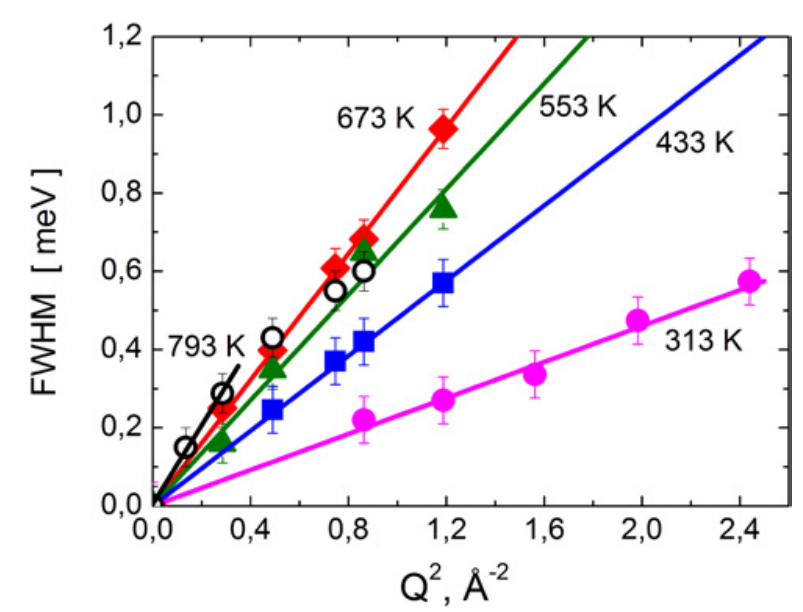

Figure 2. Incoherent QENS widths. Lines show the fit with model (5).

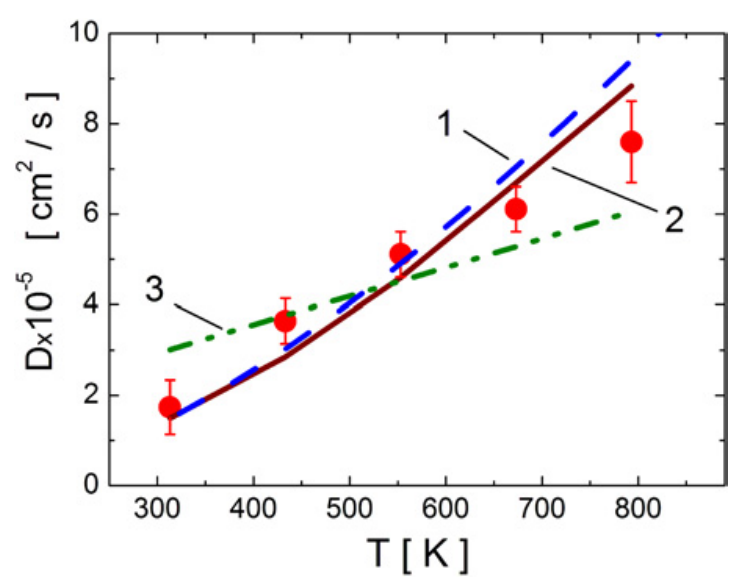

Figure 3. The self-diffusion coefficients in liquid Ga. The points represent our incoherent neutron scattering results. Line 1 shows the values obtained from the viscosity data [17]. Predictions of the hard-spheres model (2): (line 2) $\sigma$ is calculated according to (3); (line 3) $\sigma=$ const

aluminum taken from ref. [18] that were obtained from the coherent neutron scattering are also plotted. The fit with model (3) for the data of [18] gives the value $\sigma_{m} /(1-$ $0.112)=2.85 \AA$, which is close to that $(2.9 \AA)$ provided by formula (4). According to Eq. (3), the temperature dependence of $\sigma_{T}$ for $\mathrm{Ga}$ is stronger due to the lower melting point temperature.

The coherent QENS widths at $Q$ values near the maximum of $S(Q)$ are plotted in Fig. 5. In order to simplify the fitting procedure, we multiplied these widths by $S(Q)$. The experimental $S(Q)$ measured at $\mathrm{T}=326$ and $\mathrm{T}=959 \mathrm{~K}$ was taken from ref. [1] and interpolated to the temperatures of our experiment.

First, the experimental data were fitted by model (6) with $D_{E}$ and $\sigma$ as parameters. The values $D_{E}=1.46 \times$ $10^{-5} \mathrm{~cm}^{2} / \mathrm{s}$ and $\sigma=2.8 \AA$ obtained for $\mathrm{T}=313 \mathrm{~K}$ are in good agreement with the results of $[3,5]$. But the fit results derived for other temperatures seem meaningless. $D_{E}$ shows the values from $1.46 \times 10^{-5} \mathrm{~cm}^{2} / \mathrm{s}$ (at $313 \mathrm{~K}$ ) to $3.69 \times 10^{-5} \mathrm{~cm}^{2} / \mathrm{s}$ (at $793 \mathrm{~K}$ ), which are much smaller than those predicted by model (1) and obtained from the incoherent data at higher temperatures. $\sigma$ is increasing

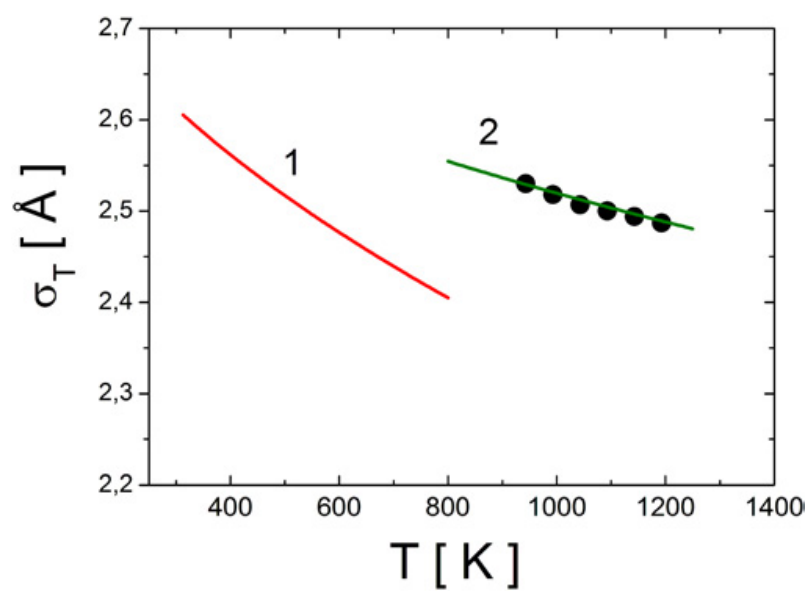

Figure 4. (1) Calculated $\sigma_{T}$ for liquid Ga. (2) Experimental $\sigma_{T}$ for liquid aluminium (points) [18] fitted with model (3) (curve).

with the temperature reaching the value of $3.03 \AA$ at $\mathrm{T}=$ $793 \mathrm{~K}$.

On the other hand, $D_{E}$ calculated using Eq. (1) with $\sigma_{T}$ given by Eq. (3) provides a very good agreement between model (6) and the experimental data but only for $Q$ values lower than that corresponding to the maximum of $S(Q)$ (Fig. 5). In other words, the non-hard-sphere effects in the liquid Ga come into play at the distances shorter than the average interatomic distance.

The main conclusion that can be done based on the analysis of our incoherent and coherent QENS data is that the longer range diffusive dynamics of liquid $\mathrm{Ga}$ is dominated by the repulsive forces between the atoms.

In order to reach a good agreement with both incoherent and coherent experimental data, one has to suppose the temperature dependence of the hard-sphere diameter $\sigma$ according to Eq. (3). However, the difference in time- and length-scales corresponding to the QENS components explored in our experiment imposes the difference in using of $D_{E}$ parameter. In order to describe the coherent line widths near the maximum of $S(Q)$, this parameter is employed as provided by Eq. (1). On the other hand, the AW correction given by Eq. (2) is important for the longer range diffusion related to the incoherent QENS at lower $Q$. This means that the microscopic dynamics observed in our coherent QENS data can be described in terms of stochastic binary collisions, as it is supposed by Enskog theory. The correlated motions should be visible at lower $Q$ region corresponding to the incoherent QENS that supposes longer length- and time-scales.

The incoherent FWHM measured at $\mathrm{T}=793 \mathrm{~K}$ shows the $Q$ dependence different from that was observed for the lower temperatures. Also, the comparison of the self-diffusion coefficients derived from the incoherent QENS with the values obtained from the viscosity data and provided by the hard-spheres model gives a reason to believe that the use of model (5) for the higher temperatures (793 K and probably $673 \mathrm{~K}$ ) results in underestimation of $D$. This could mean that our incoherent QENS data reflect the transition between different dominating mechanisms of the long range diffusion. A support for this hypothesis can be 


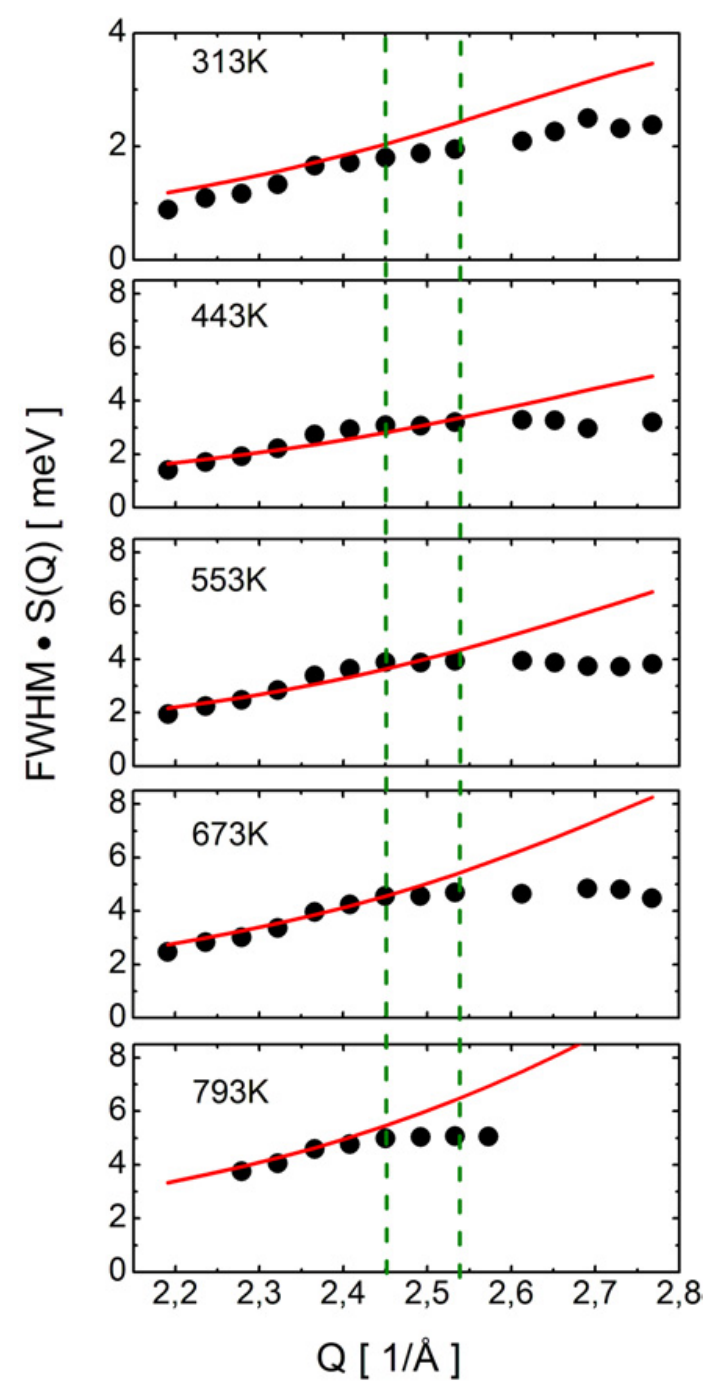

Figure 5. Experimental coherent QENS widths multiplied by $S(Q)$ (points) compared with the values calculated by the hardsphere model (curves). The $\mathrm{Q}$ region between the vertical lines corresponds to the $S(Q)$ maximum. See the text for details.

found considering AW correction values (2), which are very different for $\mathrm{T}=313 \mathrm{~K}\left(D=D_{E} \times 0.71\right)$ and $\mathrm{T}=$ $793 \mathrm{~K}\left(D=D_{E} \times 1.26\right)$ According to Ref. [6], there are two long time contributions to the diffusion process missing in the classical Enskog theory: "vortex diffusion", which originates from the self-diffusion and viscous mode coupling, and "cage diffusion" that suppose coupling the diffusive motions to the structural relaxation. Which of these two contributions dominates depends on the temperature and density of the fluid. The similar transition in the diffusive dynamics was reported for liquid $\mathrm{Na}$ [19]. Unfortunately, the accuracy of our incoherent QENS data does not allow to explore these phenomena in details, therefore another experiment with better energy resolution is needed.
The temperature dependence of the self-diffusion coefficient $D$ measured in liquid metals exhibits typically an Arrhenius-like behaviour [16,18,20,21]. Indeed, the experimental $D$ plotted in Fig. 3 can be also fitted by the Arrhenius curve with the activation energy of $\Delta E \sim 60 \mathrm{meV}$. However, in order to define, which kind of dependence (Arrhenius-type or Eq. (2)) fit the experimental $D$ better, more accurate incoherent neutron scattering data are needed. Moreover, line 2 presenting the hard-sphere model in Fig. 3 is almost indistinguishable from the Arrhenius curve with $\Delta E \sim 80 \mathrm{meV}$.

\section{References}

[1] M. C. Bellissent-Funel, P. Chieux, D. Levesque, and J. J. Weis, Phys. Rev. A 39, 6310 (1989)

[2] F. J. Bermejo, R. Fernandez-Perea, M. Alvarez et al. Phys. Rev. E 56, 3358 (1997)

[3] F.J. Bermejo, I. Bustinduy, S.J. Levett et al. Phys. Rev. B 72, 104103 (2005)

[4] L. Bove, F. Formisano, F. Sacchetti et. al. Phys. Rev. B 71, 014207 (2005)

[5] T. Scopigno, R. Di Leonardo, L. Comez, A. Q. R. Baron et al. PRL 94, 155301 (2005)

[6] E.D.G. Cohen, Physica A 194, 229 (1993)

[7] I. M. de Schepper, E.G.D. Cohen, and M. J. Zuilhof, Phys. Lett. A 101, 399 (1984)

[8] E.G.D. Cohen, P. Westerhuijs, I.M. de Schepper. Phys. Rev. Lett. 59, 2872 (1987)

[9] B. J. Alder and T. E. Wainwright, Phys. Rev. Lett. 18, 988 (1967)

[10] B. J. Alder and T. E. Wainwright, Phys. Rev. A 1, 18 (1970)

[11] B. J. Alder, D. M. Gass, and T. E. Wainwright, J. Chem. Phys. 53, 3813 (1970)

[12] J.J. Erpenbeck and W. W. Wood, Physical Review A 43, 4254 (1991)

[13] P.Protopapas, H.C.Andersen, and N.A.D.Parlee, J. Chem. Phys. 59, 15 (1973)

[14] http://flnp.jinr.ru/71/

[15] A. G. Novikov, M. N. Ivanovskii, V. V. Savostin, et al. J. Phys.: Condens. Matter 8, 3525 (1996)

[16] N.M. Blagoveschenskii, A.G. Novikov, V.V. Savostin, Physica B 407, 4567 (2012)

[17] Handbook of Chemistry and Physics, 79th ed. (CRC Press, Boca Ration, 1999)

[18] F. Demmel, D. Szubrin, W.-C. Pilgrim, C. Morkel, Phys. Rev. B 84, 014307 (2011)

[19] W.-C. Pilgrim and C. Morkel, J. Phys.: Condens. Matter 18, R585-R633 (2006)

[20] A. Meyer, S. Stüber, D. Holland-Moritz, O. Heinen, and T. Unruh, Phys. Rev. B 77, 092201 (2008)

[21] A. Meyer, Phys. Rev. B 81, 012102 (2010) 